Voix et Images

voixetimages

\title{
Le Contrat d'inversion de Michel Morin et Claude Bertrand
}

\section{Gilles Thérien}

Volume 3, numéro 2, décembre 1977

Victor-Lévy Beaulieu

URI : https://id.erudit.org/iderudit/200112ar

DOI : https://doi.org/10.7202/200112ar

Aller au sommaire du numéro

\section{Éditeur(s)}

Les Presses de l'Université du Québec

\section{ISSN}

0318-9201 (imprimé)

1705-933X (numérique)

Découvrir la revue

\section{Citer cet article}

Thérien, G. (1977). Le Contrat d'inversion de Michel Morin et Claude Bertrand. Voix et Images, 3(2), 329-330. https://doi.org/10.7202/200112ar d'utilisation que vous pouvez consulter en ligne.

https://apropos.erudit.org/fr/usagers/politique-dutilisation/ 


\section{Le Contrat d'inversion de Michel Morin et Claude Bertrand}

Je dbis faire un compte rendu. Je lis avec plaisir et puis: «Alors les égalisateurs, les uniformisateurs, les spécialistes en réduction, peut-être les trouvez-vous dans les universités ou les revues constituées." Et pan! la. page 197 vient de me mettre en demeure de me taire. Elle me définit, me circonscrit, m'interprète avant même que j'ouvre la bouche. Subitement la démarche progressive, tendue, de cet essai vient dans la chute finale me clore le bec. Et pourtant? N'ai-je pas ma part de ce contrat d'inversion, n'ai-je pas la liberté de le lire et de le dire puis de le replacer hors ou dans ma réalité? Suis-je condamné à suivre l'écriture enchevêtrée de mains doubles mais non identiques et de l'accepter comme un discours de l'institution, des éditions $\mathrm{HMH}$, d'un texte subventionné par le Conseil des arts d'Ottawa, d'un ISBN bel et bien inscrit à l'endos de la page-titre? Ce livre aurait dû alors circuler sous le manteau, porteur de la mention "top secret ".

Longtemps on s'est plu à dire et à répéter que le Québec manquait d'essayistes. A y regarder de plus près, cette affirmation est probablement très fausse. L'essai est plus fréquent qu'on ne le croit même quand il s'affable du titre modeste de roman... Le Contrat d'inversion ${ }^{1}$ est un essai, bien sûr, mais il n'est pas que cela. II est peut-être surtout une expérience d'écriture à deux mais aussi une expérience de transformation des niveaux de discours. L'habileté de l'écriture est telle qu'on parvient à croire que l'un des auteurs est de trop, sans d'ailleurs savoir lequel. L'alternance des nous, des "je", des vocatifs, qui indiquent toutes les directions et aucune, crée un climat de complicité qui associe le lecteur à cette incessante mise en scène de l'inversion.

Malgré ces dates qui ne sont les références de rien, l'histoire disparue officiellement du texte, le roman «familial", intime ou anecdotique, officiellement aboli se laisse lire dạns la négativité du texte, dans la "case vide" qui tient lieu d'origine. L'expérience personnelle accompagne, comme son double, l'expérience «philosophique» - les guillemets signifient surtout ce que j'arrive difficilement à nommer. Le dépouillement volontaire conduit à une nudité que le lecteur, placé dans la situation du voyeur, ne peut éluder. Le double, la polarisation, l'expérience des deux face à l'ordre au centre organisateur a pour effet de refouler le lecteur en ce centre, de le rendre responsable de cet ordre qu'il vit ou oppose à cette écriture qu'il lit et vit.

Le texte, magnifique par ces endroits où il devient plus charnel, laisse résonner de nombreuses harmoniques. Le Contrat puise abondamment dans un réseau intertextuel qui pointe Nietzsche, Lyotard, Deleuze, Hégel, Marx, Rousseau, etc. Ici l'expérience des philosophes "écrits" vient habiter celle des écrivants. L'absence presque totale de citations en même 
temps que la volonté marquée de lire la situation québécoise fait de ce livre un des rares exemples de volonté d'assimilation d'une tradition et de la mise en place d'un dispositif de regard authentiquement nôtre, c'est-àdire plus au sens de l'expérience personnelle qu'au sens de l'expérience nationale.

Réduire le texte à un sens? Le classer? On pourrait vouloir le faire mais cela serait difficile. Contentons-nous de suggérer une lecture, un plaisir. La poésie qui s'accommode fort bien de la rigueur des concepts déplaçables fera le reste pour le lecteur bienveillant, de même que ce vent d'anarchie qui souffle juste au moment où il est difficile de ne pas se re-présenter sous une quelconque étiquette.

Gilles Thérien

UQAM

1. Montréal, HMH, 1977. 